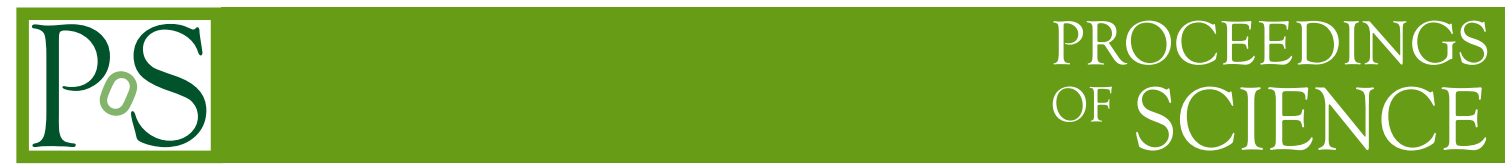

\title{
Hard processes in small systems
}

\section{Dennis V. Perepelitsa*}

University of Colorado Boulder

E-mail: dvpecolorado.edu

In these proceedings, I review the status, open questions, and prospects for measurements of high$p_{\mathrm{T}}$ processes in small collision systems. These proceedings reflect the structure and content of the talk as delivered at the High- $p_{\mathrm{T}}$ Workshop in October 2017 in Bergen, Norway.

12th International Workshop on High- $p_{\mathrm{T}}$ Physics in the RHIC/LHC Era 2-5 October, 2017

University of Bergen, Bergen, Norway

${ }^{*}$ Speaker. 


\section{Introduction}

Measurements of large transverse momentum $\left(p_{\mathrm{T}}\right)$, or hard, processes offer the opportunity to experimentally access the underlying partonic content and parton-level physics of small collision systems. For the purposes of these proceedings, systems are considered to be "small" if they do not exhibit an significant degree of jet quenching, manifesting as a modification of hard process rates or their kinematic correlations from final-state interactions in a large region of quarkgluon plasma (QGP). In addition to the traditionally studied proton+nucleus $(p+\mathrm{A})$ collisions, this includes peripheral nucleus+nucleus collisions $(\mathrm{A}+\mathrm{A})$ and ultraperipheral collisions mediated by hard photonuclear interactions $(\gamma+\mathrm{A})$.

These proceedings closely follow the structure and content of the talk given at the High- $p_{\mathrm{T}}$ Workshop in Bergen. They are thus organized around three topics: inclusive production rates of high- $p_{\mathrm{T}}$ or high-mass objects, probes of kinematics regions with novel physics effects, and understanding the onset / disappearance of jet quenching in small systems. I particularly focus on the advances in our understanding first presented at the Quark Matter 2017 (QM17) conference in Chicago [1] or soon after. Given time constraints during the talk and space constraints for these proceedings, I am not able to exhaustively discuss all relevant developments here.

\section{Inclusive production rates}

Measurements of the inclusive production of hadronic and electroweak (EW) observables via a hard scattering are a fundamental way to characterize collision systems. Production rates of fully reconstructed jets or dijets, photons, heavy EW bosons, and heavy mass quarks can each give insight into the degree of parton distribution function (PDF) modification in nuclei or constrain whether partons lose energy in the cold nuclear medium as they come into the hard scattering.

By the time of QM17, measurements of the inclusive jet production rates [2], hadron production rates [3] and jet-to-hadron fragmentation functions [4] in $5.02 \mathrm{TeV} p+\mathrm{Pb}$ collisions, now finalized and typically using $p p$ comparison data at the same energy, showed a consistent picture: when probed over a broad kinematic range, hard process rates are modified only modestly (with magnitudes similar to those expected from global nPDF analyses) and the fragmentation of partons proceeds as in vacuum. These measurements have provided new information for global nPDF fits, with Ref. [5] quantifying the impact on some widely-used modern sets.

In addition to shedding light on the fundamental partonic structure of heavy nuclei, these measurements remain important for the $\mathrm{A}+\mathrm{A}$ physics program. For example, recent measurements of jet production in 5.02 $\mathrm{TeV} \mathrm{Pb}+\mathrm{Pb}$ collisions show that suppression persists up to $p_{\mathrm{T}} \approx 1 \mathrm{TeV}$ [6]. $\mathrm{TeV}$-scale jet production at this collision energy originates from partons in the so-called EMC region $\left(x_{A}=1 \mathrm{TeV} / 2.5 \mathrm{TeV} \approx 0.4\right)$, where nuclear effects may be large. Thus constraining the role of initial state effects for this dataset, and for the larger $\mathrm{Pb}+\mathrm{Pb}$ sample to be recorded in 2018, is crucial to interpreting how these extraordinarily high- $p_{\mathrm{T}}$ jets interact with the QGP.

Electroweak bosons can provide complementary information to that given by jet and dijet production. Although they are rarer given the smaller electromagnetic (EM) and weak force coupling, they offer several advantages: (1) they can typically be measured with much better experimental control, i.e. photons and electrons in EM calorimeter sections or muons in dedicated spectrometers, 
compared to hadronic jets; (2) they feature simpler final-state topologies (e.g. direct photons do not undergo final state radiation) and thus potentially offer a better connection to the initial parton kinematics; (3) they may arise from a different mixture of quarks and gluons in the initial state; (4) they do not undergo strong final-state interactions, if any, in the system.

Two measurements in the high-statistics $p+\mathrm{Pb} 8.16 \mathrm{TeV}$ data, both new since QM17, were presented at the workshop. Isolated prompt photon production rates [7] were measured over an expansive kinematic range using $8 \mathrm{TeV} p p$ data for comparison purposes. Photon production rates were in line with the expectations from perturbative QCD calculations incorporating nPDF effects, and set an upper experimental limit on the degree of initial state energy loss. Additionally, the top quark pair production cross-section was measured through the lepton+jets channel [8] and compared to that in $p p$ collisions. This measurement, while primarily notable for observing a new fundamental particle (the top quark) in heavy ion collisions, may also have a modest impact for understanding the modification of large- $x_{\mathrm{A}}$ nuclear gluons [9].

In addition to measurements in $p+\mathrm{A}$ collisions, photonuclear processes offer a novel way to probe the partonic content of nuclei. In a typical process, the strong EM fields surrounding the fully ionized nucleus may be interpreted as an equivalent flux of quasi-real photons, one of which may fluctuate into a small quark-antiquark pair (dipole) and scatter with a gluon in the other nucleus. These events are experimentally identifiable due to their highly particular topology, in which one side of the event generally features a large rapidity gap or sum of gaps. Since there is no hadronic $\mathrm{A}+\mathrm{A}$ interaction (i.e. no color connection between the nuclei), the level of underlying event activity is highly suppressed, allowing clean measurements sensitive to nPDF effects in lower $\left(x_{\mathrm{A}}, Q^{2}\right)$ ranges than otherwise possible in hadronic collisions. The preliminary measurement of photonuclear dijet production [10] demonstrates the promise of such processes.

\section{Interesting kinematic regions}

The previous section discusses inclusive production rates in a regime where perturbative calculations based on dilute parton-parton scattering generally apply. However, in certain kinematic regions, other physical effects resulting from parton-parton or even nucleon-nucleon interactions may manifest which are not described by this picture. These include: (1) the small nuclear- $x, x_{\mathrm{A}}$, region, where the rapidly growing density of nuclear gluons may lead to non-linear QCD effects on production rates and correlations; (2) the large $x_{\mathrm{A}}$ region, where even small effects on parton kinematics may have observable consequences given the steeply falling PDF; (3) large proton- $x$, $x_{p}$, region, where novel aspects of the proton wavefunction can be studied using the nucleus as a probe of its properties, inverting the traditional concept of $p+\mathrm{A}$ collisions as a probe of nuclear effects using a well-understood proton beam. We describe each of these below.

At sufficiently low- $x_{\mathrm{A}}$ values, the rising number of gluons may cause gluon-gluon recombination processes to become important, resulting in a saturation effect which suppresses further growth of the gluon density and may have other interesting manifestations. For example, a parton from the proton may scatter coherently with a number of gluons in the nucleus, resulting in a "monojet" topology in which one jet is balanced by many soft or semi-hard particles. This effect could be explored with, for example, low- $p_{\mathrm{T}}$ central-forward dijet azimuthal correlations [11], of which a preliminary measurement was reported after the workshop [12]. 
Another novel way to access this kinematic region, not yet explored in $p+\mathrm{A}$ collisions, is through di-photon production [13]. At tree-level, two direct photons are produced in a quarkantiquark $t$-channel process. However, given the large gluon densities at LHC energies, the higher order gluon-gluon box diagram with internal quark loop contributes at a similar magnitude. Thus, a measurement of di-photon production would have similar experimental advantages as those described above for electroweak observables, and provide direct access to low- $x_{\mathrm{A}}$ nuclear gluons.

Furthermore, heavy flavor quarks at low $p_{\mathrm{T}}$ are understood to be sensitive to gluon dynamics at low- $x_{\mathrm{A}}$, since they are generally created in pairs from gluon splitting. Since QM17, new measurements of $D^{0}$ production at large-rapidity in $5.02 \mathrm{TeV}$ [14] and mid-rapidity in $8.16 \mathrm{TeV}$ [15] $p+\mathrm{Pb}$ collisions were reported. In both cases the experimental uncertainties are sub-dominant to the theoretical ones.

At large values of $x_{\mathrm{A}}$, the free nucleon PDF becomes steeply falling, resulting in a sensitivity of the production cross-section to small changes in the momentum distribution of large- $x_{\mathrm{A}}$ partons. These can be caused by the well-known Fermi motion of nucleons with respect to the center of mass of the nucleus. However, another possible source is the presence of short-range nucleon-nucleon correlations [16], in which the large relative momentum between correlated proton-neutron pairs can give rise to partons which formally have $x_{\mathrm{A}}>1$ with respect to the mean nucleon momentum [17]. Observing these so-called "superfast" quarks could offer an interesting overlap between heavy ion collisions and the so-called medium-energy nuclear physics program.

Finally, measurements in $p+\mathrm{Pb}$ collisions at the LHC [2] and $d+\mathrm{Au}$ collisions at RHIC [18] suggest that soft particle production at large nuclear-going rapidities is suppressed when one tags large- $x_{p}$ proton configurations through the final state jets. Since in $N N$ collisions this suppression does not arise from an energy conservation effect between the (forward-going) proton and soft production in the backward direction [19], one interpretation is that it reflects a "shrinking" of the proton passing through the nucleus. In this picture, proton configurations dominated by a large- $x_{p}$ parton contain fewer other partons, are physically smaller in the transverse plane, and more weakly interacting with nucleons than a proton in the average configuration [20]. Since QM17, a new global analysis of RHIC and LHC data has been performed, quantitatively extracting the change in the proton's interaction cross-section vs. $x_{p}$ [21]. A followup measurement of double parton scattering rates could further elucidate the structure of the proton in these rare configurations [22].

\section{Jet quenching in small systems}

In addition to the so-called "cold" nuclear effects described above, it is interesting to consider whether jet quenching, i.e. the energy loss of partons passing through a QGP, might be present in small systems. More generally, one can ask what is the smallest system which still exhibits the jet quenching phenomenon, and how the energy loss depends on QGP regions of arbitrarily small size.

In systematically more peripheral $\mathrm{A}+\mathrm{A}$ collisions, the nuclear modification factor, $R_{\mathrm{AA}}$, for inclusive jet or hadron spectra rises, indicating a decreasing amount of energy loss. However, in even the most peripheral events, including those with a mean number of participating nucleons or particle densities compatible with $p+\mathrm{A}$ collisions, the $R_{\mathrm{AA}}$ continues to show a suppression outside of the evaluated systematic uncertainties [23]. Global analyses which extract the path- 
length or system size dependence of energy loss based on $R_{\mathrm{AA}}$ data, including those presented at this workshop [24], suggest that there should be finite energy loss effects in $p+$ A collisions.

Since QM17, it has been proposed that the continued suppression of the jet and hadron $R_{\mathrm{AA}}$ in peripheral collisions may itself be the result of a "centrality bias" effect. Specifically, since a positive correlation between hard scattering rates and soft particle production exists in $N N$ collisions, purposefully selecting low-multiplicity events artificially suppresses hard process yields This effect is not relevant for central collisions, where hundreds of unrelated NN collisions contribute to the centrality signal, but should grow in importance in more peripheral selections. In Ref. [25], simulations with the HIJING generator and the Glauber+PYTHIA model can reproduce the suppression observed in an example peripheral bin. However, new measurements of EW boson $R_{\mathrm{AA}}$ in this data show that the expected scaling holds, in fact showing a modest enhancement in peripheral events [26]. Whether the EW measurements probe sufficiently peripheral events, or whether the same hard-soft correlations should be expected for EW as for generic hard scattering processes given the different color connections of their final states, is an open question for future investigation.

Given the difficulty in understanding a possible suppression of inclusive jet and hadron rates in small systems introduced by the multiplicity-based biases described above, jet quenching can also be searched for on a per event basis which does not rely on an absolute classification of the collision geometry. Specifically, final-state interactions should distort the inter-event momentum correlation between jets or hadrons. I focus on two examples: (1) a non-zero azimuthal anisotropy $v_{2}$ has been observed for hadrons with $p_{\mathrm{T}}=9-12 \mathrm{GeV}$ in a multiplicity selection corresponding to $0-1 \% p+\mathrm{Pb} 5.02 \mathrm{TeV}$ collisions [27]. In $\mathrm{A}+\mathrm{A}$ events, a non-zero $v_{2}$ in this kinematic region would be interpreted as a manifestation of differential (i.e. path-length dependent) energy loss. This measurement was performed using two-particle correlations, and a visible near-side correlation ridge between the high- $p_{\mathrm{T}}$ particles and soft particles can be observed. (2) However, semiinclusive (hadron-triggered) recoil jet distributions are unmodified between central and peripheral selections [28], ostensibly setting a limit on the possible amount of energy loss.

Meanwhile, the evidence for the formation of a QGP in small systems continues to mount. Shortly after the QM17 conference, a finite $v_{2}$ for $J / \psi$ particles [29] and a significant long-range near-side ridge for $D^{0}$ particles [15] in $p+\mathrm{Pb}$ collisions were reported. An obvious interpretation of these signatures is that the composition and dynamics of the system created in $p+\mathrm{A}$ collisions are qualitatively similar to the strongly-coupled, flowing medium produced in A+A collisions. These initial observations necessitate a systematic investigation of heavy flavor and quarkonia dynamics in small systems.

Searches for jet quenching have also been performed through the study of inclusive jet shapes in $p+\mathrm{Pb}$ [30], which are observed to be unmodified with respect to the vacuum reference. There has been renewed theoretical activity in imagining how jet quenching may be observed even in highmultiplicity $p p$ collisions [31]. Finally, following a common theme in this talk, energy loss effects may be measured using EW bosons as a control, i.e. in photon+jet $p_{\mathrm{T}}$ correlations. Recent measurements of fully unfolded photon+jet $p_{\mathrm{T}}$ balance distributions in $\mathrm{Pb}+\mathrm{Pb}$ and $p p$ collisions [32] have demonstrated a significant degree of experimental control over instrumental and underlying event effects, suggesting that a high-precision study could be repeated in $p+\mathrm{Pb}$ collisions. 


\section{Conclusion}

In these proceedings, the status, open questions, and prospects for measurements of high- $p_{\mathrm{T}}$ processes in small collision systems are discussed, with a focus on the developments shown at and shortly after the Quark Matter 2017 conference. I hope that progress on many of the issues raised here will be presented at the 2019 edition of the High- $p_{\mathrm{T}}$ Workshop in Knoxville, Tennessee.

\section{References}

[1] D. V. Perepelitsa, Hard processes in small systems, Nucl. Phys. A967 (2017) 113 [1707.03839].

[2] ATLAS collaboration, G. Aad et al., Centrality and rapidity dependence of inclusive jet production in $\sqrt{s_{\mathrm{NN}}}=5.02 \mathrm{TeV}$ proton-lead collisions with the ATLAS detector, Phys. Lett. B748 (2015) 392 [1412.4092].

[3] ALICE collaboration, B. B. Abelev et al., Transverse momentum dependence of inclusive primary charged-particle production in p-Pb collisions at $\sqrt{s_{\mathrm{NN}}}=5.02 \mathrm{TeV}$, Eur. Phys. J. C74 (2014) 3054 [1405.2737].

[4] ATLAS collaboration, M. Aaboud et al., Measurement of jet fragmentation in $5.02 \mathrm{TeV}$ proton-lead and proton-proton collisions with the ATLAS detector, Nucl. Phys. A978 (2018) 65 [1706.02859].

[5] N. Armesto, H. Paukkunen, J. M. Penin, C. A. Salgado and P. Zurita, An analysis of the impact of LHC Run I proton-lead data on nuclear parton densities, Eur. Phys. J. C76 (2016) 218 [1512 . 01528].

[6] ATLAS collaboration, M. Aaboud et al., Measurement of the nuclear modification factor for inclusive jets in $\mathrm{Pb}+\mathrm{Pb}$ collisions at $\sqrt{s_{\mathrm{NN}}}=5.02 \mathrm{TeV}$ with the ATLAS detector, 1805.05635 .

[7] ATLAS collaboration, Prompt photon production in $\sqrt{s_{\mathrm{NN}}}=8.16 \mathrm{TeV} p+$ Pb collisions with ATLAS, ATLAS-CONF-2017-072 (2017) .

[8] CMS collaboration, A. M. Sirunyan et al., Observation of top quark production in proton-nucleus collisions, Phys. Rev. Lett. 119 (2017) 242001 [1709.07411].

[9] D. d'Enterria, K. Krajczar and H. Paukkunen, Top-quark production in proton-nucleus and nucleus-nucleus collisions at LHC energies and beyond, Phys. Lett. B746 (2015) 64 [1501. 05879].

[10] ATLAS collaboration, Photo-nuclear dijet production in ultra-peripheral $\mathrm{Pb}+\mathrm{Pb}$ collisions, ATLAS-CONF-2017-011 (2017) .

[11] K. Kutak and S. Sapeta, Gluon saturation in dijet production in p-Pb collisions at Large Hadron Collider, Phys. Rev. D86 (2012) 094043 [1205. 5035].

[12] ATLAS collaboration, Dijet azimuthal correlations and conditional yields in $p p$ and $p+P b$ collisions at $\sqrt{s_{\mathrm{NN}}}=5.02 \mathrm{TeV}$ with the ATLAS detector, ATLAS-CONF-2018-050 (2018).

[13] ATLAS collaboration, G. Aad et al., Measurement of the isolated di-photon cross-section in pp collisions at $\sqrt{s}=7 \mathrm{TeV}$ with the ATLAS detector, Phys. Rev. D85 (2012) 012003 [1107.0581].

[14] LHCB collaboration, R. Aaij et al., Study of prompt $D^{0}$ meson production in pPb collisions at $\sqrt{s_{\mathrm{NN}}}=5 \mathrm{TeV}$, JHEP 10 (2017) 090 [1707.02750].

[15] ATLAS collaboration, D meson production and long-range azimuthal correlation in $8.16 \mathrm{TeV} p+P b$ collisions with ATLAS, ATLAS-CONF-2017-073 (2017) .

[16] O. Hen, G. A. Miller, E. Piasetzky and L. B. Weinstein, Nucleon-Nucleon Correlations, Short-lived Excitations, and the Quarks Within, Rev. Mod. Phys. 89 (2017) 045002 [1611. 09748]. 
[17] A. J. Freese, M. M. Sargsian and M. I. Strikman, Probing superfast quarks in nuclei through dijet production at the LHC, Eur. Phys. J. C75 (2015) 534 [1411.6605].

[18] PHENIX collaboration, A. Adare et al., Centrality-dependent modification of jet-production rates in deuteron-gold collisions at $\sqrt{s_{N N}}=200 \mathrm{GeV}$, Phys. Rev. Lett. 116 (2016) 122301 [1509. 04657 ].

[19] ATLAS collaboration, G. Aad et al., Measurement of the dependence of transverse energy production at large pseudorapidity on the hard-scattering kinematics of proton-proton collisions at $\sqrt{s}=2.76$ TeV with ATLAS, Phys. Lett. B756 (2016) 10 [1512.00197].

[20] M. Alvioli, B. A. Cole, L. Frankfurt, D. V. Perepelitsa and M. Strikman, Evidence for x-dependent proton color fluctuations in pA collisions at the CERN Large Hadron Collider, Phys. Rev. C93 (2016) 011902 [1409.7381].

[21] M. Alvioli, L. Frankfurt, D. Perepelitsa and M. Strikman, Global analysis of color fluctuation effects in proton- and deuteron-nucleus collisions at RHIC and the LHC, Phys. Rev. D98 (2018) 071502 [1709.04993].

[22] B. Blok, M. Strikman and U. A. Wiedemann, Hard four-jet production in pA collisions, Eur. Phys. J. C73 (2013) 2433 [1210.1477].

[23] CMS collaboration, V. Khachatryan et al., Charged-particle nuclear modification factors in PbPb and pPb collisions at $\sqrt{s_{\mathrm{NN}}}=5.02 \mathrm{TeV}$, JHEP 04 (2017) 039 [1611.01664].

[24] P. Christiansen, these proceedings.

[25] C. Loizides and A. Morsch, Absence of jet quenching in peripheral nucleus-nucleus collisions, Phys. Lett. B773 (2017) 408 [1705 . 08856$].$

[26] ATLAS collaboration, Measurement of $W$ boson production in the muon channel in $\mathrm{Pb}+\mathrm{Pb}$ collisions at $\operatorname{sqrt}(s N N)=5.02 \mathrm{TeV}$, ATLAS-CONF-2017-067 (2017) .

[27] ATLAS collaboration, G. Aad et al., Measurement of long-range pseudorapidity correlations and azimuthal harmonics in $\sqrt{s_{N N}}=5.02$ TeV proton-lead collisions with the ATLAS detector, Phys. Rev. C90 (2014) 044906 [1 409.1792$].$

[28] ALICE collaboration, S. Acharya et al., Constraints on jet quenching in $p$-Pb collisions at $\sqrt{\mathbf{S}_{\mathbf{N N}}}=$ $5.02 \mathrm{TeV}$ measured by the event-activity dependence of semi-inclusive hadron-jet distributions, Phys. Lett. B783 (2018) 95 [1712.05603].

[29] ALICE collaboration, S. Acharya et al., Search for collectivity with azimuthal J/ $\psi$-hadron correlations in high multiplicity p-Pb collisions at $\sqrt{s_{\mathrm{NN}}}=5.02$ and $8.16 \mathrm{TeV}$, Phys. Lett. B780 (2018) 7 [1709.06807].

[30] ALICE collaboration, S. Acharya et al., First measurement of jet mass in $\mathrm{Pb}-\mathrm{Pb}$ and $\mathrm{p}$-Pb collisions at the LHC, Phys. Lett. B776 (2018) 249 [1702 . 00804 ].

[31] M. L. Mangano and B. Nachman, Observables for possible QGP signatures in central pp collisions, Eur. Phys. J. C78 (2018) 343 [1708.08369].

[32] ATLAS collaboration, M. Aaboud et al., Measurement of photon-jet transverse momentum correlations in 5.02 TeV Pb+Pb and pp collisions with ATLAS, 1809.07280. 